\title{
STUDY ON OXIDATION OF PICKLE CABBAGE WASTEWATER BY SODIUM PERSULFATE
}

\author{
ZHANG, D. H. ${ }^{1}-$ GU, J. C. ${ }^{2 *}-$ OU, Y. J. ${ }^{1}-$ RAN, M. J. ${ }^{1}-$ YANG, H. M. ${ }^{1}$ \\ ${ }^{1}$ Architecture and Civil Engineering, Xihua University, 610039 ChengDu, China \\ ${ }^{2}$ Food and Biological Engineering, Xihua University, 610039 ChengDu, China \\ *Corresponding author \\ e-mail: gu6471@163.com; phone:+86-136-4809-0701
}

(Received $26^{\text {th }}$ Feb 2018; accepted $24^{\text {th }}$ May 2018)

\begin{abstract}
With activated carbon as carrier and sodium persulfate as oxidant, the preparation conditions of catalyst and the optimal reaction conditions of sodium persulfate advanced oxidation are studied. The study shows that the catalytic performance of manganese in sodium persulfate advanced oxidation treatment of pickle wastewater is the best. The optimum preparation conditions of the catalyst are as follows: at $30^{\circ} \mathrm{C}$, the carrier is soaked in $0.15 \mathrm{~mol} / \mathrm{L}$ manganese nitrate solution for $2 \mathrm{~h}$ and calcined for 2 $\mathrm{h}$ at $400{ }^{\circ} \mathrm{C}$. The optimum reaction conditions: the amount of oxidant, dosage of catalyst, reaction $\mathrm{pH}$ and reaction time are kept at $1 \mathrm{mmol} / \mathrm{L}, 10 \mathrm{~g} / \mathrm{L}, 4$ and $75 \mathrm{~min}$ respectively. and the reaction temperature is set to $30{ }^{\circ} \mathrm{C}$. Under the optimal reaction conditions, the removal rate of chemical oxygen demand (COD) can reach $84 \%$. According to the analysis of BET, FT-IR and X ray diffraction, manganese has been successfully loaded onto the carrier. Meanwhile, the activity of catalyst is affected by physical factors such as specific surface area, aperture and pore volume.
\end{abstract}

Keywords: advanced oxidation, characterization analysis, catalyzer, reaction conditions, preparation conditions, $C O D$

\section{Introduction}

As a kind of flavour food, pickle has the effect of reducing blood fat and enhancing immunity (Bao et al., 2011), and it is welcomed by the majority of consumers. With the rapid expansion of the scale of production, environmental pollution caused by a large amount of salt pickle wastewater has been aggravated. Its main pollution components are plant fiber, plant amino acids, organic acids, alcohols, salts, and inorganic salts of metals such as calcium and magnesium. Therefore, they are characterized by high chemical oxygen demand (COD), high nitrogen and phosphorus and so on. The direct discharge of pickle wastewater will pollute the local soil and water, which will cause serious eutrophication of water and salinization of the soil (Zhao et al., 2011). Therefore, the effective solution to the problem of pickle wastewater has been paid more and more attention.

At present, the methods commonly used in the treatment of high salinity, high COD are biochemical method (Wang et al., 2002; Qin et al., 2006; Chen et al., 2011), membrane separation method (Jin et al., 2009; Zhao et al., 2011) and ion exchange method (Bao et al., 2010; Yuan, 2012). The ability of microorganism to treat pollutants is reduced, because of the salt in pickle water, so the effect of traditional biochemical method is not ideal. On the basis of biological method, salt tolerant microorganism has been studied in the treatment of high salt wastewater, but there are still some shortcomings such as long taming time and the fact that it is easily affected by change of salinity (Bao et al., 2010). Ion exchange and membrane separation are ideal treatment methods for high COD and high salinity wastewater, but there is also poor resistance to 
organic pollutants, frequent membrane replacement, high treatment cost and large amount of auxiliary reagents during processing, which are likely to cause extra pollution.

The persulfate advanced oxidation technology is widely used in the field of wastewater treatment, and the strong oxidation of sulfate radical $\left(\mathrm{SO}_{4}{ }^{-}\right)$is used to deal with harmful substances in water (Wang and Cheng, 2017; Zhang, 2017; Shao et al., 2017). During the treatment, $\mathrm{S}_{2} \mathrm{O}_{8}{ }^{2-}$ is converted to $\mathrm{SO}_{4}{ }^{-} \cdot\left(\mathrm{E}_{0}=2.5 \mathrm{v}-3.1 \mathrm{v}\right)$, which is equivalent to or even higher than the redox potential of $\mathrm{HO} \cdot\left(\mathrm{E}_{0}=2.7 \mathrm{~V}-2.8 \mathrm{~V}\right)$, resulting in the oxidation and decomposition of most organic pollutants. In this paper, persulfate advanced oxidation process was used to treat the wastewater of pickled cabbage, and the optimal preparation conditions of the catalyst are explored.

\section{Materials and methods}

\section{Experimental reagents and instruments}

Reagents and materials: sulfuric acid (AR), sodium persulfate (AR), powder activated carbon, potassium dichromate (PT), mercury sulfate (AR), ammonium ferrous sulfate (AR), silver sulfate (AR), silver nitrate (AR), ferric nitrate, zinc nitrate, manganese nitrate, nickel nitrate, copper nitrate, $\mathrm{pH}$ paper, distillation water, Pickle wastewater taken from the pickle plant in Xin Fan Town, the main index of wastewater is shown in Table 1.

Table 1. Pickle wastewater quality indicators

\begin{tabular}{c|c}
\hline Project & Content \\
\hline COD & $2500 \sim 3000 \mathrm{mg} / \mathrm{L}$ \\
pH & $4.0 \sim 6.0$ \\
Turbidity & $300 \sim 400$ \\
Salt content & $0.5 \% \sim 2 \%$ \\
SS & $800 \sim 1000 \mathrm{mg} / \mathrm{L}$ \\
\hline
\end{tabular}

Equipment and instruments: electronic balance, COD125 digestion instrument, THZ82B gas bath constant temperature oscillator, DHG-9240A electrothermal constant temperature blower drying box, SZ-93 automatic double water distiller, SX2-5-12 electric furnace, constant temperature magnetic stirrer B11-2, Automatic multi station specific surface, micropore and mesoporous pore analyzer (BELSORP-max), X- ray diffractometer (EMPYREAN X), Fourier infrared spectrometer (Nicolet 6700).

\section{Method of experiment and analysis}

\section{Preparation of catalyst}

The activated carbon is placed in the beaker, soaked in distilled water, fully stirred and washed until the effluent is clarified, and then dried at $105^{\circ} \mathrm{C}$ in the oven. A certain amount of activated carbon after pretreatment was soaked in a certain concentration of nitrate solution and placed in the air bath constant temperature oscillator for a period of time. Then filtering out, and the catalyst is made by drying and calcination. 
The impregnation conditions and calcination conditions in the process of catalyst preparation were explored through experiments \#1 and \#2.

(1) Experiment \#1

At $30^{\circ} \mathrm{C}$, activated carbon, as a carrier, is immersed in different concentrations of ferric nitrate, zinc nitrate, manganese nitrate, nickel nitrate, copper nitrate for $2 \mathrm{~h}$. The calcination temperature is $400{ }^{\circ} \mathrm{C}$, and the calcination time is $2 \mathrm{~h}$. The catalyst is prepared to deal with pickle wastewater, the reaction temperature is maintained at 30 ${ }^{\circ} \mathrm{C}$, the $\mathrm{pH}$ value is about 5 , sodium persulfate dosage was $1 \mathrm{mmol} / \mathrm{L}$, reaction time is 2 h.

\section{(2) Experiment \#2}

According to the data of Experiment 1, the best immersing solution is used and immersing time is $2 \mathrm{~h}$. The calcination temperature is adjusted to 200, 300, 400, 500, $600{ }^{\circ} \mathrm{C}$, and the calcination time is $2 \mathrm{~h}$; The calcination temperature is adjusted to the best calcination temperature and the calcination time is changed from 1 to $6 \mathrm{~h}$.

\section{Method of optimizing reaction conditions}

Under the condition of normal pressure, $100 \mathrm{ml}$ pickle wastewater is placed in a conical bottle, then a certain amount of sodium persulfate and a certain amount of catalyst is added. Finally, the conical bottle is placed in a constant temperature reactor.

The reaction conditions, such as the amount of oxidant, the amount of catalyst, $\mathrm{pH}$, reaction temperature and time, were explored and optimized through experiments \#3 \#6.

(1) Experiment \#3

The optimal conditions of catalysis are determined for the treatment of pickle wastewater based on Experiment \#1 and \#2. In this part, the dosage of oxidant is changed, while the other reaction conditions are kept. In other words, the reaction temperature is maintained at $30^{\circ} \mathrm{C}$, the $\mathrm{pH}$ value is about 5 , and the reaction time is $2 \mathrm{~h}$.

(2) Experiment \#4

First, the dosage of oxidant is adjusted to be the best, then the dosage of the optimized catalyst is changed, while the other reaction conditions were maintained at 30 ${ }^{\circ} \mathrm{C}$, the $\mathrm{pH}$ value is about 5 , and the reaction time is $2 \mathrm{~h}$.

(3) Experiment \#5

First, the dosage of oxidant and catalyst are adjusted to be the best, then the $\mathrm{pH}$ value of the reaction system was changed while the other reaction conditions are maintained. (4) Experiment \#6

The experiment is carried out by adjusting $\mathrm{pH}$, oxidant dosage, catalyst dosage to the best condition. the reaction temperature and reaction time are changed from $20^{\circ} \mathrm{C}$ to $45^{\circ} \mathrm{C}$ and from $0 \mathrm{~min}$ to $120 \mathrm{~min}$. The treatment effect of wastewater was determined once every $15 \mathrm{~min}$ at different temperatures.

\section{Analysis of results}

The components of pickle wastewater is complex. The main components are plant fibre, plant amino acids, organic acids, alcohols, salts, calcium and magnesium ions etc. Therefore, in this experiment, the effect of COD removal rate was used as the evaluation index. The determination of COD was determined by potassium dichromate method (GB11914 - 89). The calculation is based on Equation 1:

$$
C O D_{\text {Removal rate }}=\frac{\left(\operatorname{COD}_{\text {inlet }}-\operatorname{COD}_{\text {effuent }}\right) \cdot 100 \%}{\operatorname{COD}_{\text {inlet }}}
$$


The specific surface area and pore size distribution of activated carbon carrier and catalyst are analyzed by BELSORP-max (full automatic multistation ratio surface, micropore and mesoporous pore analyzer).

The Nicolet 6700 infrared spectrometer was used to analyze the catalyst and the active carbon carrier, and the characteristic peak change mechanism of the material was analyzed by the EZOMNC software.

The crystal structure of catalyst and carrier activated carbon was determined and qualitatively analyzed by EMPYREAN X ray diffraction analyzer, and the variation of characteristic peak of the material was analyzed by JADE6.0 software.

\section{Results and discussion}

\section{Optimization of the catalyst preparation conditions}

Determination of active components and impregnation concentration

After the Experiment \#1, the COD value of the effluent is measured, the results are shown in Figure 1.

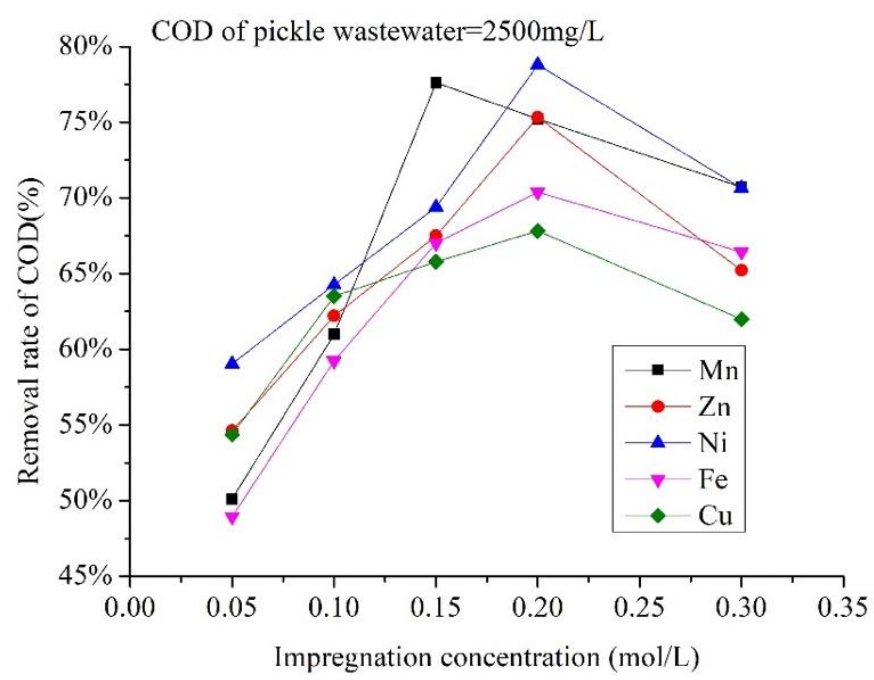

Figure 1. Treatment effect of different active component catalysts

As shown in Figure 1, the COD removal rate of all catalysts are above 35\%. In contrast, the catalytic activity of activated carbon immersed $0.2 \mathrm{~mol} / \mathrm{L}$ nickel nitrate is the highest, and the removal rate of COD reached $78.80 \%$, the second highest is the catalyst which is immersed in $0.15 \mathrm{~mol} / \mathrm{L}$ manganese nitrate, reaching $77.61 \%$. This is due to the strong adsorption capacity of activated carbon. In the process of immersion, the active component of nitrate enters its pore structure. During the reaction, the generation of $\mathrm{SO}_{4}{ }^{-}$and related chain reactions are accelerated by the transition metals in the carrier, increasing the number of $\mathrm{SO}_{4}{ }^{-}$in the reaction system, and the rate of $\mathrm{SO}_{4}{ }^{-}$ propagation is also increased. When the immersing concentration is too large, the activity of the catalyst decreases. Because in this situation, the active components in the carrier pore will be saturated. The accumulation of active components on the surface of the carrier will lead to uneven distribution, and the active sites will be obscured. The 
efforts between the catalytic efficiency of manganese and nickel is about $1 \%$, but the concentration of manganese nitrate solution is lower and the price is cheaper. Based on experimental data and economic considerations, $0.15 \mathrm{~mol} / \mathrm{L}$ manganese nitrate is used as the immersing solution, and immersing time is $2 \mathrm{~h}$.

The effect of calcination conditions on the activity of catalyst

After the Experiment \#2, the effect of calcination time and calcination temperature on the activity of the catalyst were tested. The results of the test are shown in Figure 2(a and $b$ ).

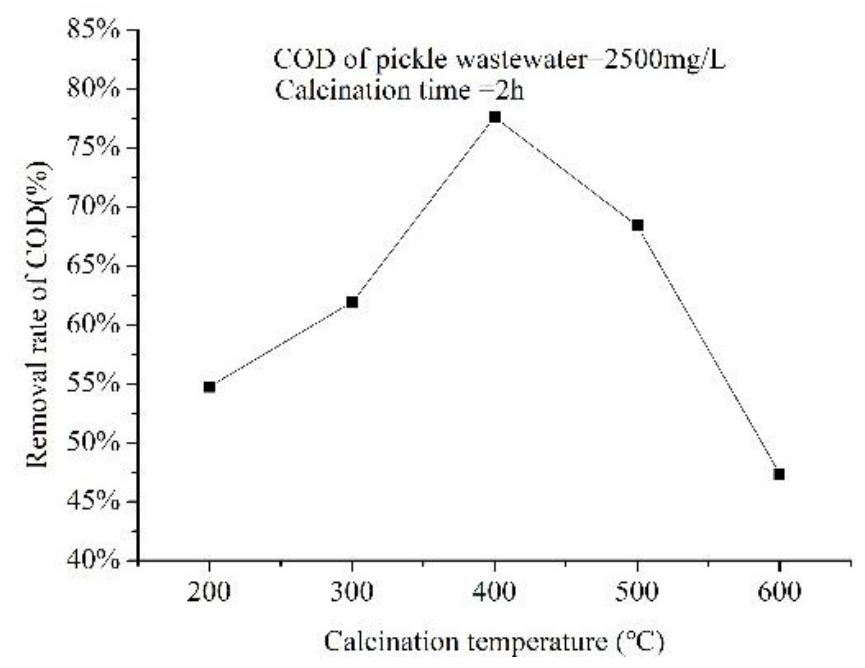

$\mathbf{a}$

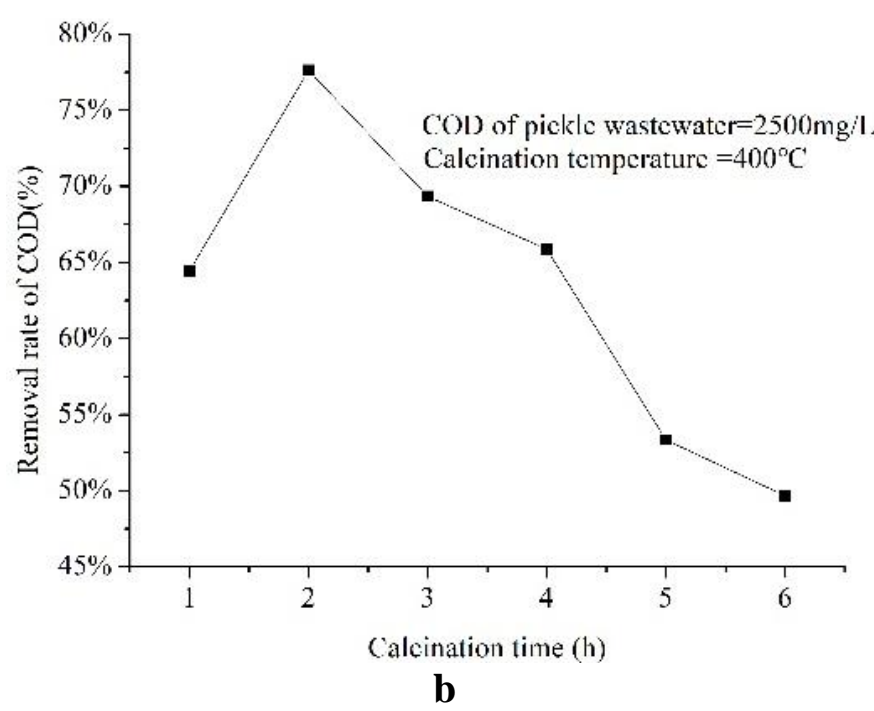

Figure 2. The effect of calcination temperature on the activity of catalyst (a). The effect of calcination time on the activity of catalyst $(b)$

As shown in Figure $2 a$, the activity of the catalyst increases with the calcination temperature when the calcination time is $2 \mathrm{~h}$ and the calcination temperature is changed during $200{ }^{\circ} \mathrm{C}$ to $400{ }^{\circ} \mathrm{C}$. This is because at a lower temperature, the nitrate on the carrier is not completely decomposed, and the activity of the catalyst is increased by 
raising the calcination temperature. The effect is best at $400{ }^{\circ} \mathrm{C}$, and the removal rate is $77.61 \%$. When the calcination temperature continues to rise, obvious sintering phenomenon will appear, and it leads to the decrease of the specific surface area and the decrease of the activity of the catalyst. From Figure $2 b$, it reveals that the calcination time is $0 \mathrm{~h}$, that is, unroasted, the treatment effect is not good. In contrast, after calcination, the activity increased significantly, This is not only due to the transformation of manganese nitrate into manganese oxide, but proper calcination can also make the surface of the carrier structure is more stable. When the calcination time is $2 \mathrm{~h}$, the removal rate of COD is the largest. The activity of catalyst decreases with increasing calcination time, because the sintering phenomenon occurs, and the pore structure collapses and the specific surface area decreases.

\section{Optimization of reaction conditions}

\section{Influence of the amount of oxidant on the treatment effect}

Through the Experiment \#3, the effect of the catalyst on the treatment effect was tested. The results of the test are shown in Figure 3.

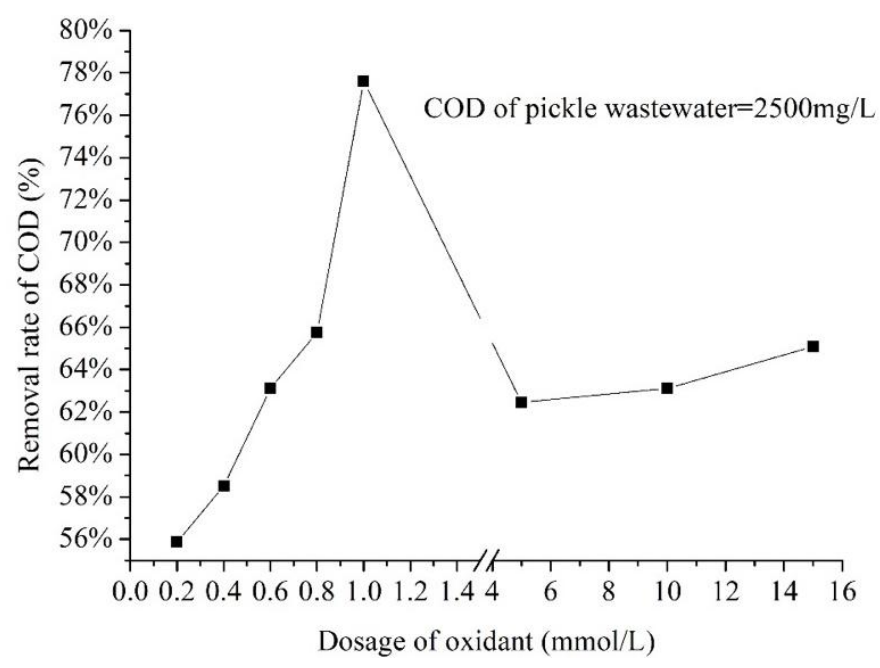

Figure 3. The effect of the amount of oxidizing agent on the treatment effect

As shown in Figure 3, with the increase of the amount of oxidant, the removal rate of COD increases first and then decreases. When the dosage is $1 \mathrm{mmol} / \mathrm{L}$, the removal rate is $77.61 \%$. When the dosage of sodium persulfate continues to increase, the removal rate of COD will decrease. When the sodium persulfate dosage is $5 \mathrm{mmol} / \mathrm{L}$, the removal rate is $62.46 \%$. The reason for this phenomenon is that with the increase of oxidants, the conversion of persulfate to $\mathrm{SO}_{4}{ }^{-}$is accelerated under the action of catalyst, and the $\mathrm{SO}_{4}^{-}$content is increased. Its strong oxidizing property is used to decompose organic matter in the waste water and the concentration of COD is reduced. When the dosage of oxidant continues to increase, in the reaction system, a large number of $\mathrm{S}_{2} \mathrm{O}_{8}{ }^{2-}$ will be quenched with $\mathrm{SO}_{4}^{-}$(Yang et al., 2010; Liang and $\mathrm{Su}, 2009$ ), which leads to the decrease of $\mathrm{SO}_{4}{ }^{-}$content. At the same time, $\mathrm{SO}_{4}{ }^{-}$will also be self coupled. The oxidant utilization is declined by these two factors. Therefore, the dosage of the oxidant in the subsequent experiment was determined to be $1 \mathrm{mmol} / \mathrm{L}$. 


\section{Influence of catalyst dosage on treatment effect}

Through the Experiment \#4, the effect of the oxidant on the reaction was tested. The results of the test are shown in Figure 4.

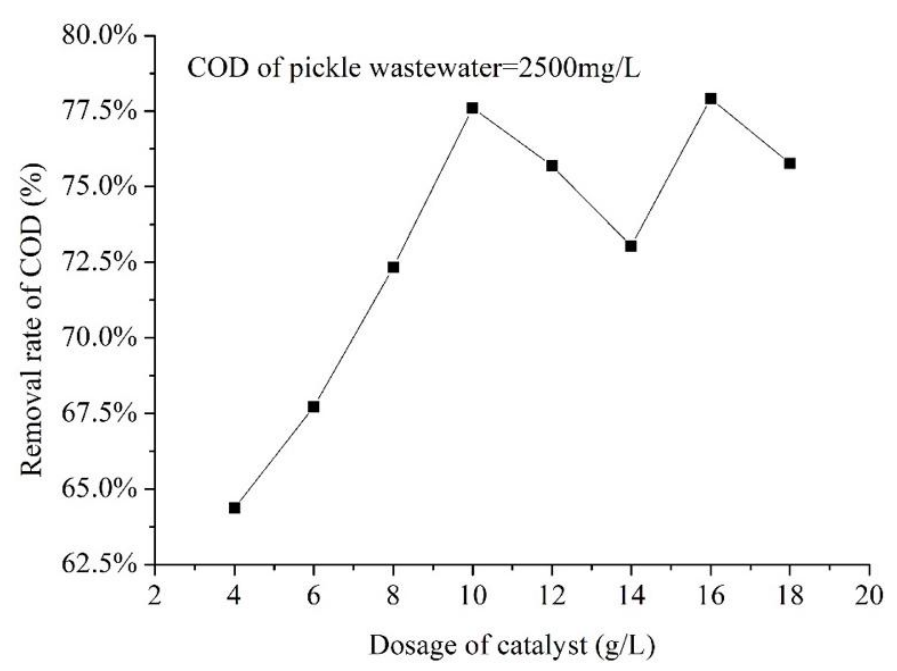

Figure 4. The effect of catalyst dosage on treatment effect

As shown in Figure 4, with the increase of the amount of catalyst, the removal rate of COD increases first and then tends to be stable. The removal rate of COD in the wastewater increases with the increase of dosage, and the removal rate is up to $77.61 \%$ when the dosage is $10 \mathrm{~g} / \mathrm{L}$. This is due to the increase of the active site with the addition of the catalyst. The contact probability of the reactant and the active site will increase. The generation of $\mathrm{SO}_{4}{ }^{-}$and the reaction of chain reaction were accelerated, resulting in the increase of $\mathrm{SO}_{4}{ }^{-} \cdot$ content, which resulted in higher reaction efficiency and increased COD removal rate. When the dosage of catalyst is more than $10 \mathrm{~g}$, the removal rate of COD always fluctuates around $75 \%$, and the change tends to be stable. When the amount of dosage is $4 \sim 18 \mathrm{~g} / \mathrm{L}$, the removal rate of COD is all above $64 \%$. Therefore, in the comprehensive economic consideration, the dosage of the subsequent experimental catalyst is determined to be $10 \mathrm{~g} / \mathrm{L}$.

\section{Influence of reaction system $\mathrm{pH}$ on reaction effect}

Through the Experiment \#5, the effect of the reaction $\mathrm{pH}$ was tested. The results of the test are shown in Figure 5: As the $\mathrm{pH}$ value of the reaction system increases, the removal rate of COD increases first and then decreases. When $\mathrm{pH}$ is 2 , the removal rate of COD is $72.34 \%$, and when $\mathrm{pH}$ is adjusted to 4 , the removal rate of COD increases to $85.81 \%$, and then continues to increase $\mathrm{pH}$ value, the COD removal rate will decrease. When $\mathrm{pH}$ is alkaline $(\mathrm{pH}>8)$, the removal rate of COD is less than $75 \%$, and when $\mathrm{pH}$ is 12 , the removal rate of COD is only $52.78 \%$. When $\mathrm{pH}$ is medium / strong alkaline $(\mathrm{pH}>8)$, the removal rate of COD is less than $75 \%$, and when $\mathrm{pH}$ is 12 , the removal rate of COD is only $52.78 \%$. It can be seen that the $\mathrm{pH}$ reaction system has great influence on the reaction effect, and this is due to the fact that the mechanism of advanced oxidation reaction system for sodium persulfate in acidic or alkaline condition 
is completely different. When $\mathrm{pH}$ is acidic, the $\mathrm{SO}_{4}{ }^{-}$in the reaction system is stable (Couttenye et al., 2002), and it can play a role in the process of oxidation.

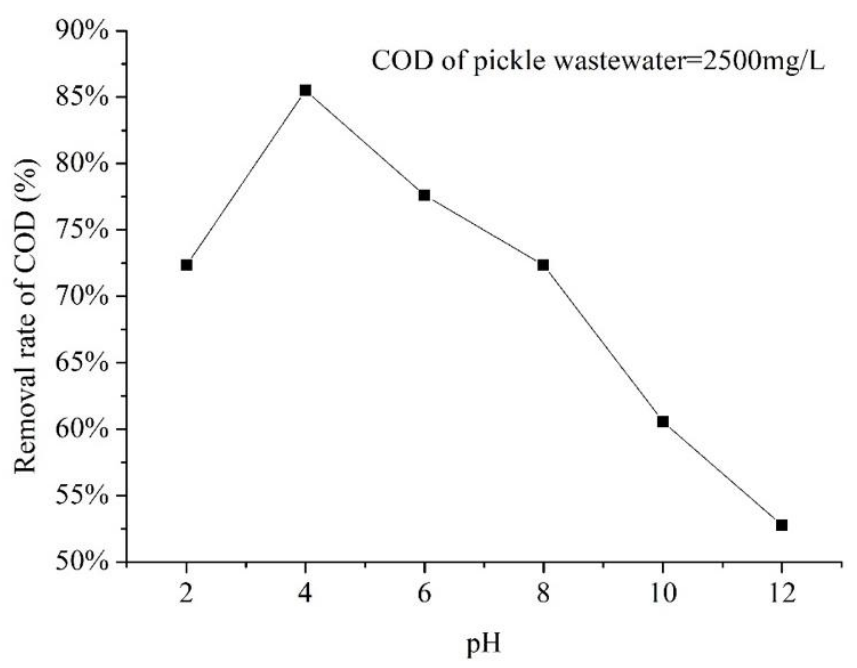

Figure 5. The effect of reaction system pH on treatment effect

When the reaction system is alkaline, in the reaction system, $\mathrm{SO}_{4}{ }^{-}$Will react with $\mathrm{H}_{2} \mathrm{O}$ and $\mathrm{OH}^{-}$, and the chemical equations are as follows:

$$
\begin{aligned}
& \mathrm{SO}_{4^{-}} \cdot+\mathrm{H}_{2} \mathrm{O} \rightarrow \mathrm{HSO}_{4^{-}}+\cdot \mathrm{OH} \\
& \mathrm{SO}_{4^{-}} \cdot+\mathrm{OH}^{-} \rightarrow \mathrm{SO}_{4}{ }^{2-}+\cdot \mathrm{OH}
\end{aligned}
$$

At this time, the hydroxyl radicals $(\cdot \mathrm{OH})$ were involved in the oxidation. However, there was a lot of $\mathrm{Cl}^{-}$in the pickle wastewater, and $\mathrm{Cl}^{-}$has quenching effect on $\cdot \mathrm{OH}$, so that $\cdot \mathrm{OH}$ have been quenched before oxidized, resulting in a sharp decrease in COD removal rate.

Based on the above experimental data and analysis, when the reaction $\mathrm{pH}$ changes during 4 to 7 , the COD removal rate is kept at a high level. In order to find the best reaction effect, the subsequent $\mathrm{pH}$ will be 4 .

\section{Influence of reaction temperature and reaction time on the treatment effect}

The results of the Experiment \#6 are shown in Figure 6: From the reaction time, the reaction in the first $15 \mathrm{~min}$ is very fast, and the COD removal rate can reach $63.69 \%$. Then, the reaction rate will slow down from 15 to $75 \mathrm{~min}$, and the reaction will tend to be stable after $75 \mathrm{~min}$. From the reaction temperature, when the temperature rises from $20{ }^{\circ} \mathrm{C}$ to $30{ }^{\circ} \mathrm{C}$, the removal rate of COD increases from about $72 \%$ to about $84 \%$, and the reaction effect is obviously better. When the temperature is higher than $30{ }^{\circ} \mathrm{C}$, the increase of temperature also plays a role in the improvement of the treatment effect but is not obvious, and the change tends to be stable. From the aspect of kinetics, when the temperature is increased, the mass transfer process between reactants and products in liquid phase and active sites is accelerated, which improves the reaction efficiency and increases the removal rate of COD. As the temperature continues to rise, the "forward" 
effect of temperature continues, but from the aspect of thermodynamics, the oxidation process of the organic matter and the adsorption process on the surface of the catalyst are both exothermic. From this point of view, the increase of temperature is not conducive to the oxidation and adsorption. Therefore, when the temperature is higher than a certain temperature, the removal rate increases little. Therefore, considering the energy saving and economy, the reaction time is $75 \mathrm{~min}$, the optimum reaction temperature is $30{ }^{\circ} \mathrm{C}$. From the experimental data, the oxidation process has a good tolerance for temperature.

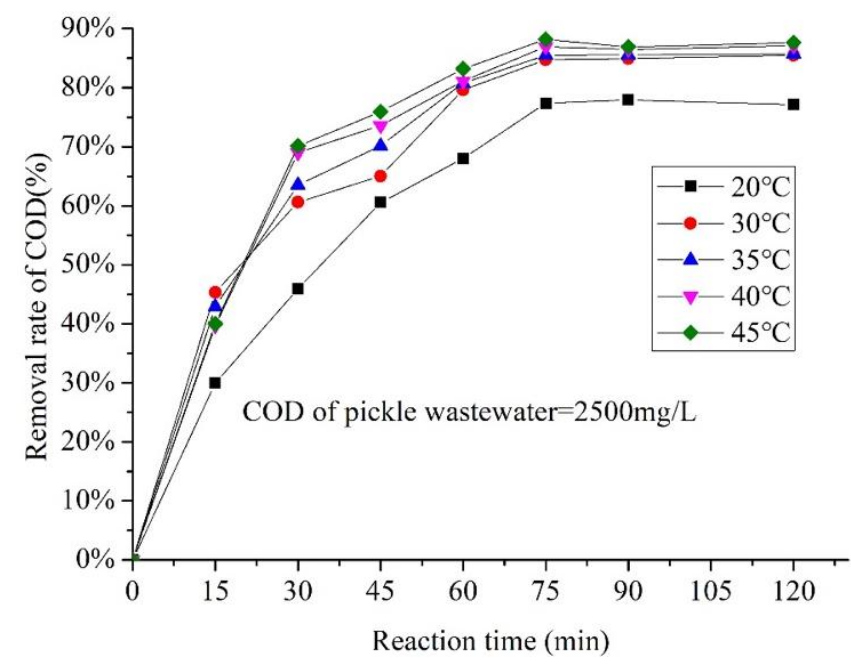

Figure 6. The effect of reaction temperature and reaction time on the treatment effect

\section{Characterization and analysis of catalyst}

\section{Specific surface area/aperture analysis}

In order to further explore the influence of immersion and calcination on the structure of the catalyst, the BET specific surface area of the prepared catalyst was tested. In the experiment, the liquid nitrogen was used as the adsorbent and the desorption curve is shown in Figure 7, and the results of the BET test are shown in Table 2.

As shown in Figure 7, the adsorption curve and the desorption curve coincide in $\mathrm{p} / \mathrm{p}$ $0<0.4$, and at $\mathrm{p} / \mathrm{p} 0>0.4$, the desorption curve is higher than the adsorption curve, then it is closed again at about $\mathrm{p} / \mathrm{p} 0=1.0$, forming a hysteresis loop. It affects the pore structure of the catalyst and is related to the network properties of the pores. According to the characteristics of this curve, the nitrogen desorption isotherm curve of the catalyst should be summed up as type IV curve, and the pore structure of the catalyst is mainly mesoporous and microporous. According to Table 2, it reveals that the specific surface area and pore structure of the carrier changed after impregnation and calcination. The specific surface area of the catalyst increases, the total volume of the micropores increases, but the average pore size and the mean pore volume decrease. This is due to the calcination which can open the inner pores of the active carbon, so the micropores are increased. But, after calcination, manganese nitrate becomes manganese oxide, and it blocks the partial pore structure of the carrier, resulting in the decrease of the average 
pore volume. And it also reflects the active components being successfully loaded onto the catalyst carrier.

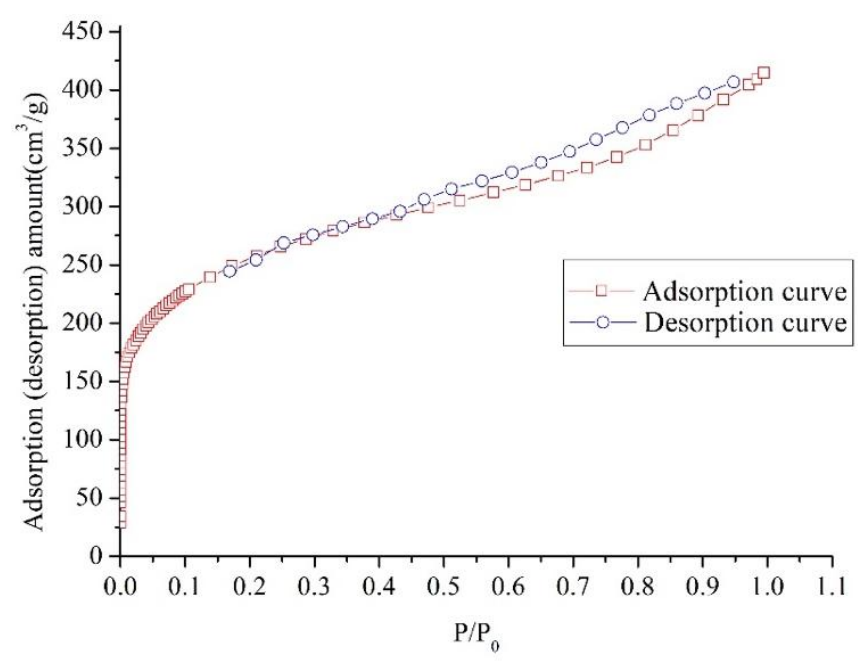

Figure 7. Catalyst for nitrogen adsorption desorption isotherm

Table 2. Catalyst BET analysis

\begin{tabular}{c|c|c|c|c|c|c}
\hline Name & $\begin{array}{c}\text { Impregnation } \\
\text { condition }\end{array}$ & $\begin{array}{c}\text { Calcination } \\
\text { condition }\end{array}$ & $\begin{array}{c}\text { Specific } \\
\text { surface area } \\
\left(\mathbf{m}^{2} / \mathbf{g}\right)\end{array}$ & $\begin{array}{c}\text { Aperture } \\
(\mathbf{n m})\end{array}$ & $\begin{array}{c}\text { Pore } \\
\mathbf{v o l u m e} \\
\left(\mathbf{c m}^{\mathbf{3}} / \mathbf{g}\right)\end{array}$ & $\begin{array}{c}\text { Total volume } \\
\mathbf{\text { of micropores }} \\
\left(\mathbf{c m}^{\mathbf{3}} / \mathbf{g}\right)\end{array}$ \\
\hline Carrier AC & -- & -- & 463.81 & 3.733 & 0.424 & 0.1874 \\
\hline $\begin{array}{c}\mathrm{Mn} / \mathrm{AC} \\
\text { catalyst }\end{array}$ & $\begin{array}{c}0.15 \mathrm{~mol} / \mathrm{L} \\
\text { manganese nitrate, } 2 \mathrm{~h}\end{array}$ & $400{ }^{\circ} \mathrm{C}, 2 \mathrm{~h}$ & 474.48 & 3.355 & 0.398 & 0.1931 \\
\hline
\end{tabular}

\section{Fourier infrared spectroscopic analysis}

Fourier infrared spectroscopy (FT-IR) was used to analyze the changes in the chemical groups of the active carbon of the carrier before and after the load. The results of the experiment are as shown in Figure 8.

Figure 8 shows that the carrier activated carbon has absorption peak at $3404.64 \mathrm{~cm}^{-1}$, $2921.88 \mathrm{~cm}^{-1}, 2844.86 \mathrm{~cm}^{-1}, 1618.52 \sim 1316.82 \mathrm{~cm}^{-1}, 1162.62 \sim 1029.14 \mathrm{~cm}^{-1}, 896.26 \mathrm{~cm}^{-}$ ${ }^{1}, 775.70 \sim 471.78 \mathrm{~cm}^{-1}$. Among them, a wider absorption peak appeared at $3404.64 \mathrm{~cm}^{-1}$, and it is caused by the hydrogen bond vibration in -OH, indicating the presence of a large number of hydroxyl groups in the samples. $2921.88 \mathrm{~cm}^{-1}$ and $2844.86 \mathrm{~cm}^{-1}$ indicate the presence of phenolic group $(\mathrm{O}-\mathrm{H})$; There are several smaller absorption peaks in the $1618.52 \sim 1316.82 \mathrm{~cm}^{-1}$ interval, which may be related to the telescopic vibration of $\mathrm{C}=\mathrm{O}$ and the presence of nitro compounds and carboxyl groups. The absorption peak in the $1162.62 \sim 1029.14 \mathrm{~cm}^{-1}$ interval indicates that there are also ethers $(=\mathrm{C}-\mathrm{O}-\mathrm{C}, \mathrm{C}-\mathrm{O}-\mathrm{C})$ in the activated carbon. The continuous and weak absorption peaks in the 500 1000 interval may be caused by the bending vibration of $-\mathrm{OH}$ and $\mathrm{C}-\mathrm{H}$. Compared with the carrier activated carbon, the overall transmittance of the $\mathrm{Mn} / \mathrm{AC}$ catalyst was reduced. Among them, the hydroxyl, carboxyl and phenolic hydroxyl groups all increased. All kinds of oxygen-containing functional groups greatly enhanced the polarity of the catalyst surface and enhanced the adsorption capacity. At the same 
time, the peak of the absorption peak of the catalyst at $516 \mathrm{~cm}^{-1}$ and $565 \mathrm{~cm}^{-1}$ is due to the telescopic vibration of the Mn-O metal bond (Li, 2015a; Li, 2015b). This shows that after the carrier activated carbon impregnated, manganese is successfully loaded, and after calcination, manganese and manganese oxides are formed.

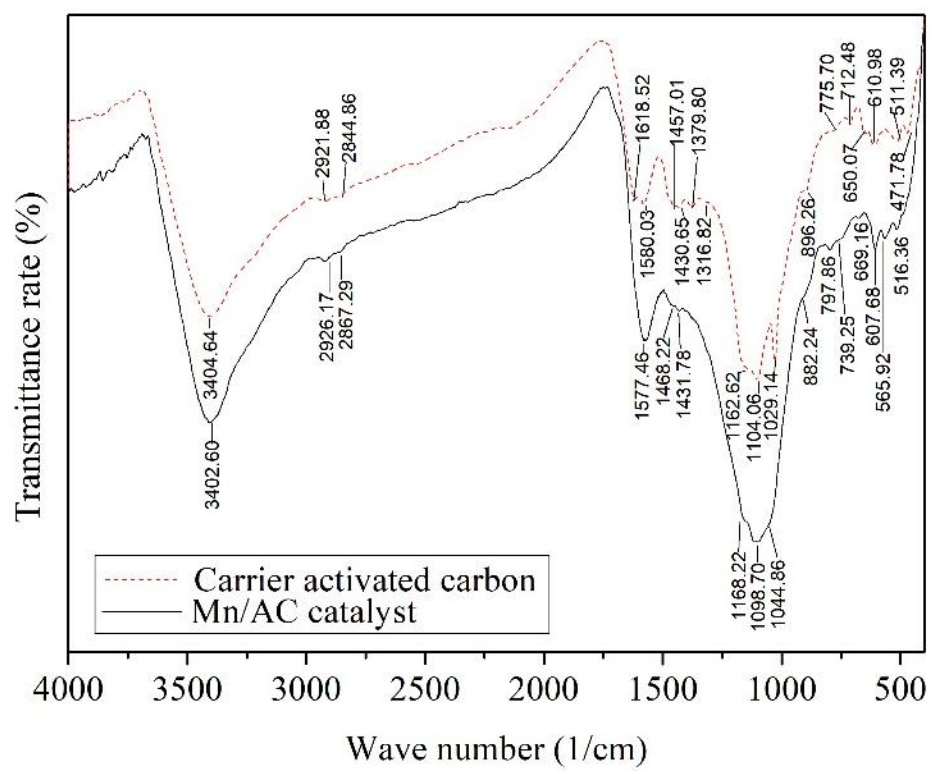

Figure 8. Comparison of infrared spectra of active carbon of carrier before and after load

\section{$X$-ray diffraction analysis}

X-ray diffraction was used to analyze the active carbon of the carrier before and after the load. By comparing the variation of the characteristic peaks, the changes in the crystals of the related material before and after the load can be found. The results of the experiment are as shown in Figure 9.

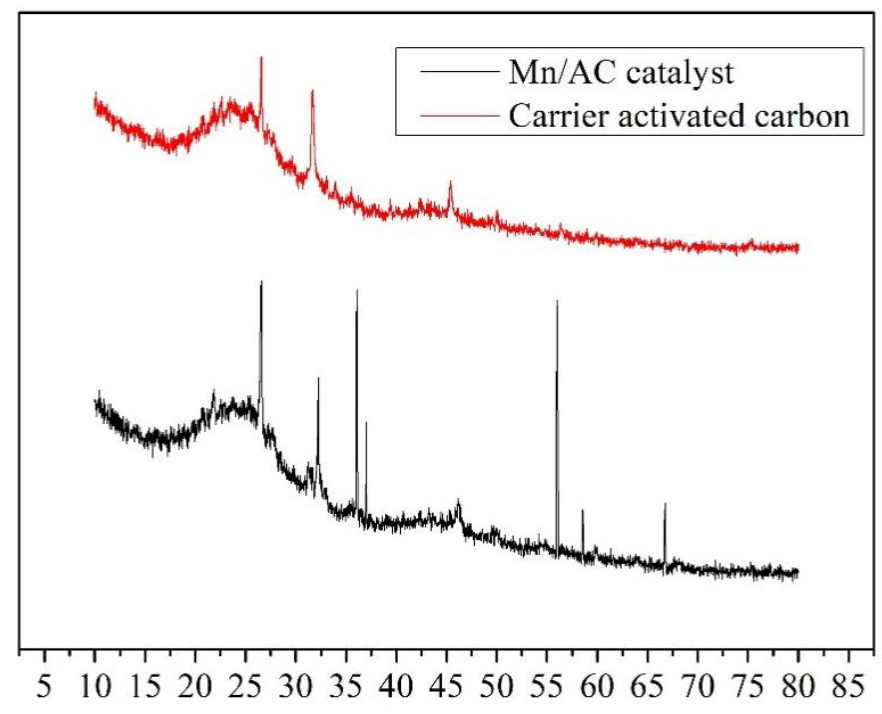

$2 \theta$

Figure 9. The Xrd pattern of activated carbon before and after load 
As shown in Figure 9, some sharp peaks of sharp diffraction are found in the carrier after treatment. The peak diffraction angle $2 \theta$ is $32.31^{\circ}, 36.09^{\circ}, 37.12^{\circ}, 56.02^{\circ}, 59.84^{\circ}$, $66.76^{\circ}$. Compared with the JCPDS standard card (30-0820), the diffraction peaks at $37.12^{\circ}, 56.02^{\circ}$, and $66.76^{\circ}$ indicate that $\mathrm{MnO}_{2}$ is formed in the carrier. Compared with the JCPDS standard card (24-0734), the diffraction peaks of $2 \theta$ at $32.31^{\circ}, 36.09^{\circ}$ and $59.84^{\circ}$ conformed to the characteristic peaks of $\mathrm{Mn}_{3} \mathrm{O}_{4}$. The characteristic peak pointed out that the manganese oxide formed the crystal, but the figure of merit (FOM) was not high, which may be related to the background peak and the sample processing conditions during the measurement. Through the comparison of the XRD diagram, it is shown that the manganese oxide has been successfully loaded with activated carbon.

\section{Conclusion}

(1) Experiments show that the manganese in sodium persulfate advanced oxidation treatment of pickle wastewater for the catalytic performance is the best. In the preparation of the catalyst, the selection of the suitable impregnation concentration can make the active component into the carrier gap and get the uniform distribution. The selection of the appropriate calcination time and temperature can efficiently convert the active component into the corresponding metal oxide, and the sintering phenomenon is controlled. Based on the experimental data, the optimum preparation conditions of the catalyst are as follows: at $30{ }^{\circ} \mathrm{C}$, the carrier is soaked in $0.15 \mathrm{~mol} / \mathrm{L}$ manganese nitrate solution for $2 \mathrm{~h}$ and calcined for $2 \mathrm{~h}$ at $400{ }^{\circ} \mathrm{C}$.

(2) The prepared catalyst was used for advanced oxidation treatment of wastewater. The effects of oxidant dosage, catalyst dosage, reaction $\mathrm{pH}$, reaction temperature and reaction time on the reaction effect were studied. The optimum reaction conditions: the dosage of oxidant is $1 \mathrm{mmol} / \mathrm{L}$, and the amount of catalyst is $10 \mathrm{~g} / \mathrm{L}$, and the reaction $\mathrm{pH}$ is 4 , and the reaction time is $75 \mathrm{~min}$, and the reaction temperature is set to $30{ }^{\circ} \mathrm{C}$. The COD can be removed $84 \%$ when the best preparation conditions of catalyst and the best reaction conditions is used in the advanced oxidation treatment of pickle wastewater.

(3) According to the BET analysis, it is found that compared with the carrier activated carbon, the specific surface area of the carrier is increased. The pore structure of the carrier material be improved by the formation of micropores. This may be the cause of the calcination. The activity of the catalyst is influenced by the specific surface area, aperture, pore volume and other physical factors.

(4) According to Fourier transform infrared spectroscopy analysis, the hydroxyl, carboxyl and phenolic hydroxyl groups on the activated carbon were increased. The oxygen functional groups greatly improve the adsorption capacity and activity of the catalyst. At the same time, the characteristic peaks of the telescopic vibration of the Mn-O metal bond were found in the catalyst. This shows that after the carrier activated carbon impregnation and calcination, the Mn has been successfully loaded.

(5) According to the $\mathrm{X}$ ray diffraction analysis, it was found that the catalyst showed a characteristic peak of $\mathrm{MnO}_{2}$ and $\mathrm{Mn}_{3} \mathrm{O}_{4}$ compared with the carrier activated carbon. This shows that the carrier activated carbon has successfully loaded the manganese element, which is in accordance with the results of the FT-IR analysis.

(6) Follow up studies can focus on the following aspects: first, further study on the regeneration of the catalyst should be carried out in order to provide a basis for actual operation and economic analysis; second, in order to apply this method to practical engineering, it is necessary to further expand the scale of experiments. 


\section{REFERENCES}

[1] Bao, S. X., Zhang, Y. M., Ding, X. T. (2010): Study on characteristics of circulating electrodialysis unit in desalination of high salinity water body. - Journal of Wuhan University of Technology 3(32): 84-87.

[2] Bao, Y., Zhang, Y., Li, H. (2011): In vitro screen of Lactobacillus plantarum as probiotic bacteria and their fermented characteristics in soymilk. - Annals of Microbiology 62(3): 1311-1320.

[3] Chen, X., Zhou, J., Gan, C. J., Li, X. P. (2011): Ultrahigh salt anaerobic biological treatment system quick start and decontamination characteristics. - Water Treatment Technology 37(6): 90-94.

[4] Couttenye, R. A., Huang, K. C., Hoag, G. E., et al. (2002): Evidence of sulfate free radical (SO4-•) formation under heat-assisted persulfate oxidation of MTBE. Proceedings of the 19th Petroleum Hydrocarbons and Organic Chemicals in Ground Water: Prevention, Assessment, and Remediation, Conference and Exposition, Atlanta, GA, United States, Nov. 5-8, 2002, pp. 345-350.

[5] Jin, K. Y., Zhou, Y., Jin, S. Y., Wang, Q., Liu, L. F. (2009): Study on the new membrane treatment of high salinity wastewater. - Water Treatment Technology 35(2): 50-53.

[6] Li, S. (2015a). Study on the Adsorption of Manganese Oxide to Nickel in Water. Chongqing University, Chongqing.

[7] Li, Z. P. (2015b). Study on the Preparation and Electrochemical Properties of $\mathrm{MnO}_{2} / \mathrm{C}$ Composites. - Xiangtan University, Xiangtan.

[8] Liang, C., Su, H. W. (2009): Identification of sulfate and hydroxyl radicals in thermally activated persulfate. - Industrial \& Engineering Chemistry Research 48(11): 472-5.

[9] Qin, Q. J., Yin, W., Lu, M., Zhang, Y. Q., Song, Z. W. (2006): SBR treatment of high salinity seafood processing wastewater. - Environmental Engineering 24(2): 79-83.

[10] Shao, Y., Xiao, L., Wu, Y. X., Wang, K. T., Zhang, Z., Wang, J. X., Wang, K. T., Wu, H. $X$. (2017): Research progress of degradation of organic wastewater by sodium persulfate. - Applied Chemical Industry 46(1): 180-183+187.

[11] Wang, M. R., Cheng, A. H. (2017): Study on degradation of phenol by manganese sulfate activated sodium persulfate. - Environmental Engineering 35(11): 62-65.

[12] Wang, Z. X., Wang, Z. Y., Hu, W. Z. (2002): Current situation and prospect of biological treatment of high salinity wastewater. - Industrial Water Treatment 22(11): 14.

[13] Yang, S. Y., Wang, P., Yang, X., Shan, L., Zhang, W. Y., Shao, X. T., Niu, R.(2010): Degradation efficiencies of azodye Acid Orange 7 by the interaction of heat, LV and anions with common Oxidants: persulfate, peroxymonosulfate and hydrogen peroxide. Journal of Hazardous Material 179(1-3): 552-558.

[14] Yuan, R. X. (2012): Experimental Study on High Temperature Anaerobic Biological Treatment of High Salinity Dyestuff Wastewater. - Donghua University, Changning Qu.

[15] Zhang, L. B., Xu, C. H., Zhu, H. N., Li, Y., Zhang, B. Z. (2017): Study on degradation of tetracycline hydrochloride by $\mathrm{Fe}^{2+} /$ sodium persulfate system. - Environmental Pollution and Control 39(7): 776-779+783.

[16] Zhao, F., Pu, B., Liu, X. Y., Chen, A. J. (2011): Treatment of pickle wastewater by PAFC and PAM complex flocculant. - Food and Fermentation Industry 37(21): 81-83.

[17] Zhao, X. G., Li, W., Zhang, Z. B., Xu, Y. H. (2011): Inhibition of liquid film rupture during falling film evaporation of high salty wastewater. - Journal of Environmental Engineering 5(4): 726-730. 